\title{
AN ARCLENGTH PROBLEM FOR CLOSE-TO-CONVEX FUNCTIONS
}

\section{P. L. DURen}

1. Let $S$ denote the class of functions

$$
f(z)=z+a_{2} z^{2}+a_{3} z^{3}+\ldots
$$

analytic and univalent in the unit disk $|z|<1$. The subclasses $C$ and $S^{*}$ of convex and starlike mappings have been extensively studied. In 1952, W. Kaplan [3] introduced the class of analytic functions $f(z)$ for which there exists $\phi \in C$, depending on $f$, with $\operatorname{Re}\left\{f^{\prime}(z) / \phi^{\prime}(z)\right\}>0$ in $|z|<1$. He called these functions close-to-convex, and proved that every such function is univalent. We shall denote by $K$ the normalized class of close-to-convex functions. The classes are related by the proper inclusions $C \subset S * \subset K \subset S$.

For $f \in S$ and $0<r<1$, let

$$
A_{r}(f)=\iint_{|\varepsilon| \leqslant r}\left|f^{\prime}(z)\right|^{2} d x d y=\pi \sum_{n=1}^{\infty} n\left|a_{n}\right|^{2} r^{2 n} \quad\left(a_{1}=1\right)
$$

and

$$
L_{r}(f)=\int_{|z|=r}\left|f^{\prime}(z)\right||d z|
$$

denote, respectively, the area of the image of the disk $|z| \leqslant r$ and the arclength of the image of the circle $|z|=r$. The extremal problems

$$
\begin{gathered}
\max _{f \in S} A_{r}(f) \\
\max _{f \in S} L_{\boldsymbol{r}}(f)
\end{gathered}
$$

are apparently unsolved. Because of the Bieberbach conjecture $\left|a_{n}\right| \leqslant n$, with equality only for the Koebe function

$$
k(z)=z /(1-z)^{2}=z+2 z^{2}+3 z^{3}+\ldots
$$

or one of its rotations, one strongly suspects that the Koebe function solves problem (1). Were the Bieberbach conjecture proved, this would be an immediate corollary. Since the Bieberbach conjecture has been settled for close-to-convex functions [8], $k(z)$ maximizes $A_{r}(f)$ among all $f \in K$. At any rate, the inequality $\left|a_{n}\right|<e n$ gives

$$
A_{r}(f)<\frac{e^{2} \pi r^{2}\left(1+4 r^{2}+r^{4}\right)}{\left(1-r^{2}\right)^{4}}, \quad f \in S .
$$

Received 7 January, 1964.

[JournaI London MATH. Soc., 39 (1964), 757-761] 
As for problem (2), A. Marx [7] proved that $k(z)$ maximizes $L_{r}(f)$ within the class $S^{*}$ of starlike mappings. One might doubt that $k(z)$ is the solution within the full class $S$, because there would seem to be too little control over oscillation. However, we shall show that $k(z)$ remains extremal in $K$, the class of close-to-convex functions. There is other evidence that $k(z)$ may maximize $L_{r}(f)$ within $S$. It is not difficult to prove $[6 ;$ p. 215$]$ that

$$
L_{r}(f) \leqslant \frac{2 \pi r(1+r)}{(1-r)^{2}}
$$

for all $f \in S$. But, as we shall point out,

$$
L_{r}(k)>\frac{\pi r(1+r)}{2(1-r)^{2}} .
$$

For bounded starlike functions, F. R. Keogh [5] has proved

$$
L_{r}=O\left(\log \frac{1}{1-r}\right) \text {, }
$$

and W. K. Hayman [2] has shown this result to be best possible. In $C$, $L_{r}(f)$ is maximized by $f(z)=z /(1-z)[4]$.

Theorem 1. For each $r(0<r<1)$, and for every close-to-convex function $f \in K, L_{r}(f) \leqslant L_{r}(k)$. Equality can occur only for $f(z)=e^{i \alpha} k\left(e^{-i \alpha} z\right)$, $\alpha$ real.

The proof depends upon a Herglotz type representation formula for close-to-convex functions. At the end of the paper we evaluate $L_{r}(k)$ in terms of standard elliptic integrals.

2. Let $\mathscr{P}$ denote the class of functions $P(z)$ analytic and satisfying $\operatorname{Re}\{P(z)\}>0$ in $|z|<1$, with the normalization $P(0)=1$. Each $P \in \mathscr{P}$ may be represented by the Herglotz formula

$$
P(z)=\int_{0}^{2 \pi} \frac{1+z e^{i l}}{1-z e^{i t}} d \mu(t),
$$

where $\mu(t)$ is non-decreasing and has total variation 1 on $0 \leqslant t \leqslant 2 \pi$. As is well known, $f \in S^{*}$ if and only if $z f^{\prime}(z) / f(z) \in \mathscr{P}$. Thus (3) and integration with respect to $z$ yield the representation

$$
\log \frac{f(z)}{z}=\int_{0}^{2 \pi} \log \left[\frac{e^{-i t} k\left(z e^{i l}\right)}{z}\right] d \mu(t)
$$

for starlike functions. This has an interesting interpretation. The set of all functions $\log [f(z) / z], f \in S^{*}$, is a convex set whose extreme points correspond to the rotations of the Koebe function.

Since $\phi(z) \in C$ if and only if $z \phi^{\prime}(z) \in S^{*}$, the close-to-convex functions are precisely those functions $f(z)$ for which

$$
f^{\prime}(z)=P(z) \psi(z) / z, \quad P \in \mathscr{P}, \quad \psi \in S^{*} .
$$


Hence, to every $f \in K$ there corresponds a pair of non-decreasing functions $\nu(s)$ and $\mu(t)$, each of total variation 1 , such that

$$
f^{\prime}(z)=\int_{0}^{2 \pi} \frac{1+z e^{i s}}{1-z e^{i s}} d \nu(s) \cdot \exp \left\{\int_{0}^{2 \pi} \log \left[\frac{1}{\left(1-z e^{i i}\right)^{2}}\right] d \mu(t)\right\} .
$$

We remark that the starlike functions are the subclass for which $\nu=\mu$.

By the continuous form of the arithmetic-geometric mean inequality,

$$
\left|f^{\prime}(z)\right| \leqslant \int_{0}^{2 \pi}\left|\frac{1+z e^{i s}}{1-z e^{i s}}\right| d \nu(s) \int_{0}^{2 \pi} \frac{d \mu(t)}{\left|1-z e^{i t}\right|^{2}} .
$$

Thus

$$
\begin{aligned}
L_{r}(f) & =r \int_{0}^{2 \pi}\left|f^{\prime}\left(r e^{i \theta}\right)\right| d \theta \\
& \leqslant r \int_{0}^{2 \pi} d \nu(s) \int_{0}^{2 \pi} d \mu(t) \int_{0}^{2 \pi}\left|\frac{1+r e^{i(\theta+s)}}{1-r e^{i(\theta+s)}}\right| \frac{d \theta}{\left|1-r e^{i(\theta+\theta)}\right|^{2}} \\
& \leqslant r \max _{0 \leqslant l \leqslant 2 \pi} I(t),
\end{aligned}
$$

where

$$
I(t)=\int_{0}^{2 \pi}\left|\frac{1+r e^{i \theta}}{1-r e^{i \theta}}\right| \frac{d \theta}{\left|1-r e^{i(\theta+\theta)}\right|^{2}} .
$$

The proof of Theorem 1 is completed by establishing the following lemma.

Lemma. $\quad I(t)<I(0), 0<t<2 \pi$.

Proof. Let $u(\theta)=\left|\frac{1+r e^{i \theta}}{1-r e^{i \theta}}\right|$. One finds

$$
I^{\prime}(t)=2 r \int_{0}^{n} \frac{\sin x[u(x+t)-u(x-t)]}{\left[1-2 r \cos x+r^{2}\right]^{2}} d x .
$$

It is clear that $I^{\prime}(0)=0$. On the other hand,

$$
[u(\theta)]^{2}=\frac{1+\rho \cos \theta}{1-\rho \cos \theta}, \quad \rho=\frac{2 r}{1+r^{2}} .
$$

A short calculation gives

$$
[u(x+t)]^{2}-[u(x-t)]^{2}=-\frac{4 \rho \sin x \sin t}{[1-\rho \cos (x+t)][1-\rho \cos (x-t)]} .
$$

Division by $\{u(x+t)+u(x-t)\}$ shows that the integrand in (5) is negative for $0<t<\pi$, positive for $\pi<t<2 \pi$. Thus $I^{\prime}(t)<0,0<t<\pi ; I^{\prime}(t)>0$, $\pi<t<2 \pi$. Hence the maximum value is $I(0)=I(2 \pi)$.

3. For completeness, we shall now evaluate $L_{r}(k)$ in terms of the standard elliptic integrals $K=K(\rho)$ and $E=E(\rho)$ of the first and second kinds, with modulus $\rho$. 
THEOREM 2. With $\rho=2 r /\left(1+r^{2}\right)$,

$$
L_{r}(k)=2 \rho\left[\frac{2 E(\rho)}{1-\rho^{2}}-K(\rho)\right]>\frac{\pi r(1+r)}{2(1-r)^{2}}
$$

Proof.

$$
L_{r}(k)=r \int_{0}^{2 \pi} \frac{\left|1+r e^{i \theta}\right|}{\left|1-r e^{i \theta}\right|^{3}} d \theta=\rho \int_{0}^{\pi} \frac{(1+\rho \cos \theta)^{\frac{1}{2}}}{(1-\rho \cos \theta)^{\frac{3}{2}}} d \theta .
$$

Setting $x=\cos \theta$ for $0 \leqslant \theta \leqslant \pi / 2$ and $x=-\cos \theta$ for $\pi / 2 \leqslant \theta \leqslant \pi$, one obtains

$$
L_{r}(k)=2 \rho \int_{0}^{1} \frac{1+\rho^{2} x^{2}}{1-\rho^{2} x^{2}} \frac{d x}{\left[\left(1-x^{2}\right)\left(1-\rho^{2} x^{2}\right)\right]^{\frac{1}{2}}} .
$$

This can be evaluated by means of $[1 ; 219.07,315.02,219.04,318.02]$, with the result as given above.

To estimate $L_{r}(k)$ from below, note $[1 ; 710.03]$ that $E$ is a concave function of $\rho^{2}$, so

$$
E(\rho)>\rho^{2}+\frac{1}{2} \pi\left(1-\rho^{2}\right) .
$$

Together with the trivial estimate $K(\rho)<\frac{1}{2} \pi\left(1-\rho^{2}\right)^{-t}$, this gives (after a calculation)

$$
\frac{(1-r)^{2}}{r(1+r)} L_{r}(k)>\frac{2 \pi+(32-8 \pi) r^{2}+6 \pi r^{4}}{(1+r)^{3}\left(1+r^{2}\right)}>\frac{2 \pi\left(1+r^{2}+3 r^{4}\right)}{(1+r)^{3}\left(1+r^{2}\right)} .
$$

To prove this is greater than $\frac{1}{2} \pi$ for $0<r<1$, we must show

$$
4\left(1+r^{2}+3 r^{4}\right)>(1+r)^{3}\left(1+r^{2}\right),
$$

or $3-3 r-4 r^{3}+9 r^{4}-r^{5}>0$.

But this last expression is greater than

$$
3(1-r)-4 r^{3}(1-2 r) \text {. }
$$

This is evidently positive for $r \geqslant \frac{1}{2}$; for $r<\frac{1}{2}$, it is

$$
>\frac{3}{2}-4 r(1-2 r) \geqslant \frac{3}{2}-4\left(\frac{1}{8}\right)=1>0 \text {. }
$$

The constant $\frac{1}{2} \pi$ can surely be improved by more careful estimation.

It is easily seen that

$$
L_{r}(k) \sim \frac{4}{(1-r)^{2}} \quad(r \rightarrow 1)
$$

\section{References}

1. P. F. Byrd and M. D. Friedman, Handbook of elliptic integrals for engineers and physicists (Springer-Verlag, Berlin, 1954).

2. W. K. Hayman, "On functions with positive real part", Journal London Math. Soc., 36 (1961), 35-48.

3. W. Kaplan, "Close-tọ-convex schlicht functions", Michigan Math. J., 1 (1952), 169-185, 
4. F. R. Keogh, "Some inequalities for convex and ster-shaped domains", Journal London Math. Soc., 29 (1954), 121-123.

5. F. R. Keogh, " Some theorems on conformal mapping of bounded star-shaped domains ", Proc. London Math. Soc., 9 (1959), 481-491.

6. J. E. Littlewood, Lectures on the theory of functions (Oxford University Press, 1944).

7. A. Marx, "Untersuchungen über schlichte Abbildungen", Math. Annalen, 107 (1932), 40-67.

8. M. O. Reade, "On close-to-convex univalent functions", Michigan Math. J., 3 (1955), 59-62.

University of Michigan,

Ann Arbor, Michigan, U.S.A. 